\title{
Otitis media causes and management
}

\section{Ziyad Aljohani ${ }^{1}$, Abdulaziz Alghonaim², Rayhanah Alhaddad ${ }^{3}$, Wadiah ALShaif ${ }^{3}$, Rahaf AIThomali ${ }^{4}$, Ali Asiry ${ }^{5}$, Lujain Hefni ${ }^{4}$, Sarah Nagadi ${ }^{4}$, Roba Taha ${ }^{6}$}

\author{
${ }^{1}$ Ibn Sina National College for Medical Studies, Jeddah, KSA \\ ${ }^{2}$ Almaarefa Colleges for Science \& Technology, Riyadh, KSA \\ ${ }^{3}$ Imam Abdulrahman Bin Faisal University, Dammam, KSA \\ ${ }^{4}$ King Abdulaziz University, Jeddah, KSA \\ ${ }^{5}$ King Khalid University, Abha, KSA \\ ${ }^{6}$ Taibah University, Al Madinah, KSA
}

Received: 23 July 2018

Accepted: 10 August 2018

\section{*Correspondence:}

Dr. Ziyad Aljohani,

E-mail: Zeyad.jehani@gmail.com

Copyright: (C) the author(s), publisher and licensee Medip Academy. This is an open-access article distributed under the terms of the Creative Commons Attribution Non-Commercial License, which permits unrestricted non-commercial use, distribution, and reproduction in any medium, provided the original work is properly cited.

\begin{abstract}
Otitis media is a major cause of health care visits across the world, and its complications are significant causes of preventable hearing loss, predominantly in the developing world. They are a group of inflammatory and complex infective conditions that affect the middle ear. They have several different subtypes, and affects primarily children from 3 to 7 years of age. When not treated properly, they can lead to many complications including permanent hearing loss. In this article we will discuss updates on recent scientific developments in the field of otitis media clinical management and research. We conducted this review using a comprehensive search of MEDLINE, PubMed and EMBASE from January 1979 to March 2017. The following search terms were used: otitis media, middle ear infections, pediatric infections, causes of otitis media, treatment otitis media, and prevention of otitis media. Otitis media is one of the most common pathologies in the pediatric age group making it one the most common reasons of doctor visits. Fortunately, the management is simple and promising, but if neglected, can lead to several complications including permanent hearing loss. Newer studies are focusing on preventive measures such as pneumococcal vaccine, and in avoiding creation of resistant species of the common bacteria that cause the pathology.
\end{abstract}

Keywords: Otitis media, Middle ear pathology, Pediatric infections, Microbiology otitis media, Management pediatric infection

\section{INTRODUCTION}

Otitis media $(\mathrm{OM})$ is a group of inflammatory and complex infective conditions that affect the middle ear. They can be classified as many subtypes based on the differences in presentation, treatment, and associated complications. OM is a major cause of health care visits across the world, and its complications are significant causes of preventable hearing loss, predominantly in the developing world. In this article we will discuss updates on recent scientific developments in the field of OM clinical management and research. ${ }^{1}$

$\mathrm{OM}$ is a common pathology of the middle ear and its mucosa, behind the tympanic membrane. The middle ear is a cavity that contains the ear ossicles which consist of malleus, incus, and stapes, along with the with the eustachian tube placed anteriorly directing to the nasopharynx, posteriorly to the mastoid air cells, laterally the tympanic membrane, and medially to the inner ear. Other vital nearby structures are the brain and meninges superiorly and the sigmoid sinus in the posterior, and 
therefore, any infection of the middle ear can spread to surrounding structures causing serious results. The lining of the middle ear is by modified respiratory epithelium, which includes ciliated cells and goblet cells. This epithelium produces mucins which are transported down the eustachian tube. ${ }^{2}$

A relatively common complication of acute otitis media (AOM) is acute mastoiditis which is acute inflammation of the air cells and mastoid periosteum. Contrary to AOM and acute mastoiditis, OM with effusion (OME) is a chronic inflammatory condition which classically affects children between ages 3 to 7 years old. OME is less prevalent in adults and when it occurs, it is often an indication of other underlying diagnoses. ${ }^{3}$

The yearly prevalence of OM diagnoses in the United States of America dropped by 28\% from 1997 and 2007, where it affected 247 per 1,000 children (compared to 345 earlier). This decline is accredited to the introduction of the pneumococcal conjugate vaccine, which gave the impression to have decreased the number of individuals suffering from OM as reported in several studies. ${ }^{4}$

\section{METHODS}

\section{Data sources and search terms}

We conducted this review using a comprehensive search of MEDLINE, PubMed and EMBASE, from January 1979 to March 2017. The following search terms were used: otitis media, middle ear infections, pediatric infections, causes of otitis media, treatment otitis media, and prevention of otitis media

\section{Data extraction}

Two reviewers have independently reviewed the studies, abstracted data and disagreements were resolved by consensus. Studies were evaluated for quality and a review protocol was followed throughout.

This study was done after approval of review board of King Abdulaziz University.

\section{DISCUSSION}

\section{Causes}

\section{Microbiology}

OM has a multifactorial etiology and is related to anatomical variations, cell biology of the middle ear cleft (middle ear cavity, mastoid, eustachian tube) and nasopharynx, as well as the pathophysiology including the interaction between microbial agents and resulting host immune response..$^{5}$ Viral upper respiratory tract infections frequently precede or coincide with AOM episodes; few examples consist of respiratory syncytial virus, cytomegalovirus, and adenovirus. Viral infections are now believed to have a central role in AOM progression, and recent randomized controlled trials have validated the role of antiviral treatment for AOM. It is alleged that viral infection of the nasopharynx produces an environment that supports bacterial colonization, adhesion to cells, and eventually invasion of the middle ear. ${ }^{6}$

The bacterial pathogens that commonly cause upper respiratory tract infections are also those most frequently isolated from middle ear effusions in AOM. They are Streptococcus pneumoniae, Moraxella catarrhalis, Haemophilus influenzae, and less commonly Staphylococcus aureus, and Streptococcus pyogenes; they are speculated to enter the middle ear cleft through the eustachian tube. There is a inconstant incidence of detecting these bacteria from effusions in AOM, but those most frequently detected are $S$. pneumoniae and $H$. influenzae in recent studies. ${ }^{7}$ Similar bacteria have also been found in cultures from fluid in OME, even though different studies in dissimilar circumstances in various countries may yield different findings. At large, the bacteria in OME are parallel to those detected in recurrent AOM (RAOM). ${ }^{8}$

Upper respiratory tract infections can result in mucosal congestion in the nasopharynx and the eustachian tube. The subsequent congestion precludes normal eustachian tube function, as well as pressure regulation is altered within the middle ear. If unresolved, nasopharyngeal pathogens can get aspirated into the middle ear. The presence of these pathogens then encourages inflammation, leading to pus collection within the middle ear, causing the clinical symptoms of AOM. For the duration of this inflammatory period, the middle ear ossicles become less mobile and can be cause to undergo resorption, which could even bring about permanent conductive hearing loss ${ }^{[9]}$. Studies have demonstrated that patients with smaller mastoid cavities have a greater risk of getting chronic middle ear disease; nevertheless, whether this effect is contributory is still debatable. Secondary to ototoxicity, patients with chronic infection have a risk of developing sensorineural hearing loss. ${ }^{6}$

\section{Genetic predisposition}

The genetic factors that predispose to $\mathrm{OM}$ are not well spoken about, however a few genetic targets have been acknowledged. Inheritance of AOM and CSOM estimating around $40 \%-70 \%$ has been reported; but, the majority of genes underlying this predisposition are yet to be recognized. ${ }^{10}$ It is probable that there are elements of innate defense molecules which could become defective, leading to greater OM susceptibility. Therefore, some potential therapeutic targets are the genes that regulate expression of mucin, production of mucus, and response of host to bacteria in the middle ear. More studies about the genetics of OM could also lead us to developing preventative measures, or minimalize risk factors in vulnerable individuals. ${ }^{11}$ 
Research in genetics has also acknowledged the important role for hypoxia in OME pathology, and this can partially explain the effectiveness of ventilation tubes, as it relieves hypoxia in the middle ear.

\section{Diagnosis}

There are several guidelines to help physicians in the diagnosis of $\mathrm{OM}$ and its subtypes. AOM is distinguished from OME and CSOM on the basis of thorough history and examination findings. The gold standard for the diagnosis of middle ear fluid is considered myringotomy, which is surgical excision of the ear drum; however, it is unpractical to indicate every child to this surgical procedure, whereas the diagnosis can be made based upon the assessment in the clinic. ${ }^{5}$

Clinically, AOM is a purulent middle ear process, for that reason the signs and symptoms consistent with acute inflammation are usually found. AOM classically has a short history, and is generally associated with otalgia, fever, otorrhea, irritability, lethargy, vomiting, and anorexia; however, the symptoms alone have low sensitivity and specificity for establishing diagnosis. The American Academy of Pediatrics guidelines recommend that AOM must be diagnosed in children presenting with moderate to severe bulging of the tympanic membrane, or presenting with new onset otorrhea which is not secondary to otitis externa. ${ }^{12}$ The diagnosis can also be made when there is mild bulging of the tympanic membrane accompanied with otalgia or severe tympanic membrane erythema. The nonexistence of a middle ear effusion, which is assessed with pneumatic otoscopy or tympanometry makes the diagnosis is unlikely. Pneumatic otoscopy and tympanometry evaluate the mobility of the ear drum to conclude effusion presence. If a non-perforated ear drum is not mobile, that indicates that a middle ear effusion is present; both techniques depend on changing the ear canal pressure, with pneumatic otoscopy picturing the ear drum directly and tympanometry measuring mobility by using sound reflection. The condition is defined as ROAM if a patient has three episodes of AOM within a six-month period, or four in one year. ${ }^{13}$

OME can either happen as a lingering effect of AOM, or without any preceding history. In children, clinical manifestations can include a history of hearing difficulties, attention deficits, behavioral problems, speech delay and delayed language development, clumsiness, and suboptimal balance. Otoscopy is crucial in making the definite diagnosis, with sensitivity and specificity reaching up to $90 \%$ and $80 \%$, respectively. ${ }^{14}$ This may be further enhanced by using pneumatic otoscopy. The clinical findings can vary, and include abnormal color ranging from yellow to amber to blue; retracted or concave tympanic membrane; and presence of air-fluid levels. Additional evidence is taken with an audiogram, including hearing testing which can show mild conductive hearing loss and tympanogram that can demonstrate an immobile ear drum with negative middle ear pressure. Parental concerns regarding hearing loss can be unreliable and must be confirmed with a formal audiological investigation. ${ }^{5}$

CSOM is diagnosed after a permanent tympanic perforation is identified together with middle ear mucositis with or without continuous otorrhea. The discharge should be noted for a minimum of two to six weeks. Therefore history is crucial in differentiating between CSOM, otitis externa, and AOM. In CSOM pain is not generally a dominant feature and ear discharge is expected to last a longer duration. The diagnosis is established with otoscopy which will frequently notice a tympanic membrane perforation associated with middle ear discharge. ${ }^{15}$

\section{Treatment}

Well established recommendations exist for the medical and surgical management of different types of OM.

Generally, AOM follows a promising course when treated with appropriate analgesia and anti-pyretic, without antibiotic treatment. Meta-analyses have revealed that around $80 \%$ of children experience spontaneous relief of AOM within 2 days to 2 weeks. In children with age less than 2 years, such results are more variable and resolution of OM may be as low as $30 \%$ in a few days. The overall high rates of resolution indicate that simple observation may be appropriate in children diagnosed with AOM in the absence of other suspected complications. ${ }^{16}$

Current guidelines in USA for the treatment of AOM endorse that antibiotics must be used in children aged over 6 months when AOM is severe, unilateral or bilateral. Sever AOM is defined as temperature of $39^{\circ} \mathrm{C}$ or more, moderate to severe otalgia, or otalgia lasting at least 48 hours. ${ }^{17}$ Antibiotics should also be given if AOM is not very severe, but it is bilateral in a child of age of 623 months. In the case of mild and moderate unilateral AOM in a child aged 6-23 months, or mild and moderate unilateral/bilateral AOM in a child aged 2 years or older, antibiotics can be suggested or only observed. If observation is chosen, a method should be planned to give antibiotics in case symptoms do not improve in 2-3 days. In addition to evaluation of the child's clinical condition, the thoughts of parents or caregivers must be assessed and decisions must be made jointly. Pain management and assessment is an important aspect of treating AOM. It is worth noting that an initial period of observation is not associated with a greater complication risk when compared to those children receiving immediate antibiotic treatment. ${ }^{16,18}$

The empiric prescription of antibiotics for treating AOM ranges from $31 \%$ in the Netherlands to $98 \%$ in the Australia and USA. Amoxicillin remains the antibiotic of choice unless the child has taken this antibiotic in the past 
30 days or is suffering from coexisting purulent. Antibiotics with added beta-lactamase cover must be used in such cases, or when there is evidence of RAOM, or a history of AOM refractory to amoxicillin. Patient allergies must also be considered and alternative agents should be used in situations where penicillin allergy is recorded. $^{19}$

In case of RAOM, surgically inserted ventilation tubes must be advised if RAOM is accompanied with a continuous middle ear effusion in between AOM attacks. Prophylactic antibiotics are usually not suggested for RAOM, although a systematic review study has noted that they can be beneficial. However, due to concerns about exposing to antibiotics for long duration and their potential side effects, ventilation tubes commonly end up being the favorable option. Ventilation tubes are only effective for the duration of their stay time. Most ventilation tubes can stay up to 6-9 months after placement. $^{20}$

Similar to AOM, many children with OME do not need treatment because of high number of spontaneous resolution. Nevertheless, if OME is bilateral and persistent for more than three months, the probability of natural resolution becomes much lower and offering treatment can be beneficial. Current guidelines of USA and UK suggest a 3-month duration for observation with making assessment of the degree of hearing loss, its impact on a child's development, and serial audiometry before deciding the need for treatment. ${ }^{21}$ Guidelines suggest either hearing aids or surgery for putting ventilation. Ventilation tube insertion is concomitant with a number of risks, such as purulent otorrhea (10\%-26\%), retraction pockets (21\%). Myringosclerosis (39\%-65\%) and persistent tympanic membrane perforations $(3 \%){ }^{22}$ Additionally, once tubes are removed OME may return. One trial of short-term tubes showed that around $25 \%$ of children needed another set of ventilation tubes within 2 years. It is believed that adenoidectomy may play a role in preventing recurrent OME, however, due to accompanying risks it is often not recommended as a primary management of OME, unless there are persistent, or recurrent upper respiratory tract infections. Several other management plans for OME have been devised, including steroid, antibiotics and antihistamines, but are not at present, recommended. ${ }^{3}$

Unlike OME and AOM, the ultimate management for CSOM is often surgical, with several different techniques designed to repair the ear drum and control infection. Conservative management is favored in certain patient groups, with the goal of reducing attacks of recurrent infection and discharge and is consequently associated with hearing loss. The most conservative approach is serial aural toilet after that the use of antibiotics, topical steroids, and antiseptics. ${ }^{23}$ Topical quinolones, such as ciprofloxacin, have been noted to be the most beneficial treatment in a recent review, however, although these are licensed inside the USA, they are, at present, not licensed as ear drops in some other countries, including the UK. Many of the commonly used ear drops are formulations based on aminoglycosides, and there are concerns about their possible ototoxicity adverse effect when used in the perforated tympanic membrane, the current agreement is that their use is safe in short term, strictly supervised courses, and ultimately less ototoxic than the infection itself. Conservative management on its own is normally chosen based on patient preferences, if patient has surgical contraindications, the non-affected ear has hearing loss, or when the risks of surgery compensate its benefit. $^{24}$

\section{Preventive measures}

AOM is a common reason for antibiotic use in pediatric age group, and the most common cause for surgery in children in the developed world is the management of OME and AOM with ventilation tube insertion. Yet antibiotic use may lead to emergence of resistance and side effects, whilst ventilation tubes usually require a general anesthetic, extrude after a period of time, and many children need repeat surgery. Better treatment of AOM and OME would therefore be welcome. The ideal management is prevention; therefore current research is focusing on achieving this target. ${ }^{25}$

\section{Pneumococcal vaccine}

Preventative measures for the development and progression of OM are important in order to limit the impact of this disease, side effects from associated antibiotic use, and therefore the emerging bacterial resistance. The antigens for vaccination have been identified as $S$. pneumoniae being the commonest cause of AOM, M. catarrhalis and nontypeable $H$. influenza. The pneumococcal conjugate vaccine was initially developed to control invasive pneumococcal disease such as pneumonia, but later proved to be useful in targeting the most common cause of AOM, and hence has attracted a lot of recent attention.

Several systematic reviews have established the effectiveness of pneumococcal vaccine in preventing pneumococcal AOM. $^{26}$ After administration of the 7valent pneumococcal conjugate vaccine (PCV7), the incidence of AOM in children declined in North America. In infants, a reduction of $43 \%$ in AOM, $42 \%$ in antibiotic prescription, and $32 \%$ in AOM-related costs was witnessed. ${ }^{27}$ Studies in Canada have shown an inclusive 25.2\% decline in AOM from 2000 to 2007, where vaccine was the cause in $13.2 \%$, and showed the largest impact in infants age group. Substantial declines in nasopharyngeal vaccine serotypes were also noted after introduction of PCV7. While PCV7 caused decrease of serotypes that were covered by the vaccine, pneumococcal serotype 19A increased. Unfortunately, isolates of S. pneumoniae serotype 19A are resistant to all US Food and Drug Administration approved drugs that are used in children to treat AOM. ${ }^{28}$ 
Regardless of promising results, there are concerns regarding the long-term benefit from immunization. After introducing vaccination, AOM-related complications including mastoiditis as well as other intracranial complications decreased; nevertheless, they surprisingly returned to levels of pre-vaccination period after only a few years. This fall and subsequent rise was also been recorded in pneumococcal infections infecting other sites, however in general these infections tend to be less commonly prevalent compared to trends before vaccination was introduced. Replacement of serotype is a concern due to potential resistance, similarly as in case of non-pneumococcal pathogens. ${ }^{29}$

A possible method of overcoming resistance to vaccine would be formulating vaccine using broadly conserved protein antigens; this could be independent of serotypes and supposedly not associated with replacement of serotype. This form of vaccine is potentially more affordable and mainly convenient in developing countries, where their need is greatest. More recent pneumococcal vaccines that can protect against additional serotypes have been developed, including the 11-valent pneumococcal polysaccharide conjugate vaccine with protein $\mathrm{D}$ as a carrier and the 13-valent pneumococcal vaccine, which may also possess further benefit against nontypeable $H$. influenzae $\mathrm{OM}^{30}$

\section{CONCLUSION}

Otitis media is one of the most common pathologies in the pediatric age group making it one the most common reasons of doctor visits. Fortunately, the management is simple and promising, but if neglected, can lead to several complications including permanent hearing loss. Newer studies are focusing on preventive measures such as pneumococcal vaccine, and in avoiding creation of resistant species of the common bacteria that cause the pathology.

Funding: No funding sources Conflict of interest: None declared

Ethical approval: Not required

\section{REFERENCES}

1. Monasta L, Ronfani L, Marchetti F, Montico M, Vecchi Brumatti L, Bavcar A, et al. Burden of disease caused by otitis media: systematic review and global estimates. PLoS One. 2012;7:e36226.

2. Bluestone CD, Doyle WJ. Anatomy and physiology of eustachian tube and middle ear related to otitis media. J Allergy Clin Immunol. 1988;81:997-1003.

3. Qureishi A, Lee Y, Belfield K, Birchall JP, Daniel M. Update on otitis media - prevention and treatment. Infect Drug Resist. 2014;7:15-24.

4. Liese JG, Silfverdal SA, Giaquinto C, Carmona A, Larcombe JH, Garcia-Sicilia J, et al. Incidence and clinical presentation of acute otitis media in children aged $<6$ years in European medical practices. Epidemiol Infect. 2014;142:1778-88.

5. Rovers MM, Schilder AG, Zielhuis GA, Rosenfeld RM. Otitis media. Lancet. 2004;363:465-73.

6. Murphy TF, Chonmaitree T, Barenkamp S, Kyd J, Nokso-Koivisto J, Patel JA, et al. Panel 5: Microbiology and immunology panel. Otolaryngol Head Neck Surg. 2013;148:64-89.

7. Pumarola F, Mares J, Losada I, Minguella I, Moraga F, Tarrago D, et al. Microbiology of bacteria causing recurrent acute otitis media (AOM) and AOM treatment failure in young children in Spain: shifting pathogens in the post-pneumococcal conjugate vaccination era. Int $J$ Pediatr Otorhinolaryngol. 2013;77:1231-6.

8. Stol K, Verhaegh SJ, Graamans K, Engel JA, Sturm PD, Melchers WJ, et al. Microbial profiling does not differentiate between childhood recurrent acute otitis media and chronic otitis media with effusion. Int J Pediatr Otorhinolaryngol 2013;77:488-93.

9. Salomonsen RL, Hermansson A, Caye-Thomasen P. Ossicular bone modeling in acute otitis media. Otol Neurotol. 2010;31:1109-14.

10. Rye MS, Bhutta MF, Cheeseman MT, Burgner D, Blackwell JM, Brown SD, et al. Unraveling the genetics of otitis media: from mouse to human and back again. Mamm Genome. 2011;22:66-82.

11. Li JD, Hermansson A, Ryan AF, Bakaletz LO, Brown SD, Cheeseman MT, et al. Panel 4: Recent advances in otitis media in molecular biology, biochemistry, genetics, and animal models. Otolaryngol Head Neck Surg. 2013;148:52-63.

12. Lieberthal AS, Carroll AE, Chonmaitree T, Ganiats TG, Hoberman A, Jackson MA, et al. The diagnosis and management of acute otitis media. Pediatrics. 2013;131:964-99.

13. Whittemore KR, Jr. What is the role of tympanostomy tubes in the treatment of recurrent acute otitis media? Laryngoscope. 2013;123:9-10.

14. Bluestone CD, Cantekin EI. Design factors in the characterization and identification of otitis media and certain related conditions. Ann Otol Rhinol Laryngol Suppl. 1979;88:13-28.

15. Marchisio P, Chonmaitree $\mathrm{T}$, Leibovitz E, Lieberthal A, Lous J, Mandel E, et al. Panel 7: Treatment and comparative effectiveness research. Otolaryngol Head Neck Surg. 2013;148:102-21.

16. Glasziou PP, Del Mar CB, Sanders SL, Hayem M. Antibiotics for acute otitis media in children. Cochrane Database Syst Rev. 2004;(1):CD000219.

17. American Academy of Pediatrics Subcommittee on Management of Acute Otitis Media. Diagnosis and management of acute otitis media. Pediatrics. 2004; 113:1451-65.

18. Buyukcam A, Kara A, Bedir T, Gulhan B, Ozdemir $\mathrm{H}$, Sutcu $\mathrm{M}$, et al. Pediatricians' attitudes in management of acute otitis media and ear pain in Turkey. Int J Pediatr Otorhinolaryngol. 2018;107:14-20. 
19. Sakulchit T, Goldman RD. Antibiotic therapy for children with acute otitis media. Can Fam Physician. 2017;63:685-7.

20. Rosenfeld RM, Schwartz SR, Pynnonen MA, Tunkel DE, Hussey HM, Fichera JS, et al. Clinical practice guideline: Tympanostomy tubes in children. Otolaryngol Head Neck Surg. 2013;149:1-35.

21. Daniel M, Kamani T, El-Shunnar S, Jaberoo MC, Harrison A, Yalamanchili S, et al. National Institute for Clinical Excellence guidelines on the surgical management of otitis media with effusion: are they being followed and have they changed practice? Int J Pediatr Otorhinolaryngol. 2013;77:54-8.

22. Vlastarakos PV, Nikolopoulos TP, Korres S, Tavoulari E, Tzagaroulakis A, Ferekidis E. Grommets in otitis media with effusion: the most frequent operation in children. But is it associated with significant complications? Eur J Pediatr. 2007;166:385-91.

23. Macfadyen CA, Acuin JM, Gamble C. Systemic antibiotics versus topical treatments for chronically discharging ears with underlying eardrum perforations. Cochrane Database Syst Rev. 2006;(1):CD005608.

24. Phillips JS, Yung MW, Burton MJ, Swan IR. Evidence review and ENT-UK consensus report for the use of aminoglycoside-containing ear drops in the presence of an open middle ear. Clin Otolaryngol. 2007;32:330-6.

25. Argaw-Denboba A, Abejew AA, Mekonnen AG. Antibiotic-Resistant Bacteria Are Major Threats of
Otitis Media in Wollo Area, Northeastern Ethiopia: A Ten-Year Retrospective Analysis. Int J Microbiol. 2016;2016:8724671.

26. Pelton SI, Pettigrew MM, Barenkamp SJ, Godfroid F, Grijalva CG, Leach A, et al. Panel 6: Vaccines. Otolaryngol Head Neck Surg. 2013;148:E90-101.

27. Zhou F, Shefer A, Kong Y, Nuorti JP. Trends in acute otitis media-related health care utilization by privately insured young children in the United States, 1997-2004. Pediatrics. 2008;121:253-60.

28. Pichichero ME, Casey JR. Emergence of a multiresistant serotype 19A pneumococcal strain not included in the 7-valent conjugate vaccine as an otopathogen in children. JAMA. 2007;298:1772-8.

29. Daniel M, Gautam S, Scrivener TA, Meller C, Levin B, Curotta J. What effect has pneumococcal vaccination had on acute mastoiditis? J Laryngol Otol. 2013;127(1):30-4.

30. Levine OS, Cherian T, Hajjeh R, Knoll MD. Progress and future challenges in coordinated surveillance and detection of pneumococcal and Hib disease in developing countries. Clin Infect Dis. 2009;48(2):33-6.

Cite this article as: Aljohani Z, Alghonaim A, Alhaddad R, ALShaif W, AlThomali R, Asiry A, et al. Otitis media causes and management. Int $\mathrm{J}$ Community Med Public Health 2018;5:3703-8. 